\title{
REATOR ELETRÔNICO PARA ILUMINAÇÃO FLUORESCENTE BOOST PUSH-PULL COM ALTO FATOR DE POTÊNCIA EMPREGANDO UM ÚNICO INTERRUPTOR
}

\author{
F. E. Bisogno, \\ A. R. Seidel, \\ T. B. Marchesan, \\ R. N. do Prado \\ GEDRE - PPGEE - NUPEDEE - UFSM \\ 97105-900 Santa Maria - RS \\ Brasil
}

\begin{abstract}
Resumo - Este artigo descreve um reator eletrônico que opera com um único estágio de conversão de potência empregando apenas um interruptor ativo, com alto fator de potência para duas lâmpadas fluorescentes T1240W. A topologia emprega um conversor boost no modo de condução descontínuo para a correção do fator de potência. $O$ estágio inversor utiliza o conversor push-pull que faz a alimentação em alta freqüência do filtro LCC ressonante e das lâmpadas. Os princípios de operação, equações de projeto e os resultados experimentais são obtidos para uma freqüência de operação de $50 \mathrm{kHz}$ e tensão de alimentação de 110V RMS, $60 \mathrm{~Hz}$.
\end{abstract}

Abstract - This paper describes a high power factor electronic ballast employing a single power converting stage and a single power switch. The converter provides a high-frequency to supply the lamp. High power factor is achieved through a boost converter operating in a discontinuous conduction mode as a pre-regulator stage. The inverter stage employs a push-pull converter that supplies the LCC filter and Lamps. Operating principles, design equations and experimental results are obtained for the following: $50 \mathrm{kHz}$ switching frequency, $110 \mathrm{~V}$ RMS line voltage, two T1240 W fluorescent lamps and $60 \mathrm{~Hz}$ line frequency.

\section{INTRODUÇÃO}

Trabalhar com reatores eletrônicos em alta freqüência é fundamental para economizar energia em função da melhor eficiência luminosa $(\mathrm{Lm} / \mathrm{W})$ [1], [2]. A alta frequiência faz com que os reatores eletrônicos sejam mais leves, menores, sem ruído audível e proporcionem uma maior vida útil à lâmpada, ao contrário dos reatores eletromagnéticos convencionais operando em $60 \mathrm{~Hz}$, que requerem um dispositivo de grande volume para limitar a corrente [3].

As lâmpadas fluorescentes que operam com reatores eletromagnéticos em $60 \mathrm{~Hz}$ apresentam ondulação de tensão a freqüência de $120 \mathrm{~Hz}$ com $33 \%$ de decréscimo da luminosidade [4], [5] [9].

Os reatores eletrônicos com correção de fator de potência operam com dois estágios de conversão, sendo o primeiro o estágio de correção do fator de potência e o segundo o estágio de inversão.

Para a correção do fator de potência geralmente são utilizados os conversores boost, flyback e buck-boost. No estágio inversor são geralmente utilizados os conversores half-bridge e push-pull [6], [7], [8], [10]. A topologia proposta possui um fator de potência elevado, alimentando a lâmpada fluorescente em alta freqüência através de um único estágio de conversão, utilizando os conversores boost no modo de condução descontínuo e push-pull, empregando um único interruptor ativo.

\section{TOPOLOGIA PROPOSTA}

A configuração básica da topologia proposta é mostrada na Fig. 1, composta por uma fonte de tensão monofásica $V_{i n}$, ponte retificadora formada pelos diodos $D_{I}-D_{4}$, um conversor boost, um inversor push-pull, dois filtros de alta freqüência e duas lâmpadas fluorescentes $\operatorname{Lamp}_{1}$ e Lamp 2 .

O conversor boost empregado para correção do fator de potência é composto pelo indutor $L_{B o o s t}$, um interruptor ativo $S$ e um capacitor $C_{\text {out }}$.

O estágio de inversão é constituído pelo conversor pushpull, formado pelos indutores $L_{P 1}, L_{P 2}$, o diodo $D_{5}$ e o interruptor ativo $S$, o qual é compartilhado com o conversor boost.

O filtro de EMI é empregado na entrada para eliminar as harmônicas de alta freqüência e é formado pelo indutor $L_{i n}$, e o capacitor $C_{i n}$. Na saída é empregado o filtro LCC sérieparalelo ressonante que faz a alimentação em alta freqüência das lâmpadas, sendo constituído por $L_{o u}, C_{P 1}, C_{P 2}$ e $C_{S}$.

\section{PRINCÍPIOS DE OPERAÇÃO}

O comportamento do circuito proposto é analisado considerando duas seções: a seção de alta frequiência (lado da carga) e a seção de baixa frequiência (lado da fonte) que é simples e não é descrita.

A seção de alta frequiência é dividida em três estágios de operação que são descritos a seguir.

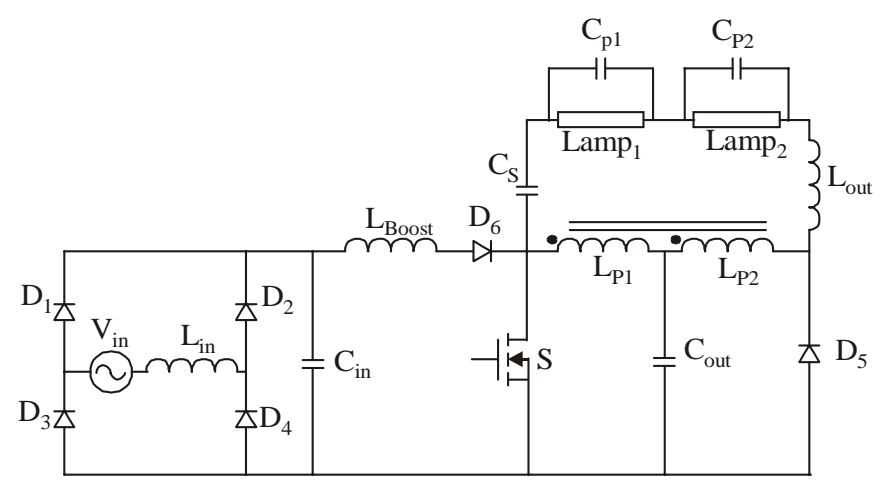

Fig. 1. Topologia proposta 


\section{A. Primeiro Estágio}

$\mathrm{O}$ circuito e seu equivalente simplificado do primeiro estágio são mostrados na Fig. 2.(a) e (d) respectivamente. O estágio é caracterizado pela condução do interruptor ativo $S$ e pela polarização reversa do diodo $D_{5}$. Neste estágio a fonte de tensão $V_{i n}$ fornece energia para o indutor $L_{B o o s t}$ determinando o crescimento linear da corrente $i_{\text {Lboost }}$, considerando que a tensão $V_{\text {in }}$ permanece aproximadamente constante durante um período de comutação. $\mathrm{O}$ capacitor $C_{\text {out }}$ é aproximado por uma fonte de tensão constante $V_{o}$ que é aplicada ao indutor $L_{P 1}$ e refletida pelo acoplamento ao indutor $L_{P 2}$, resultando em uma tensão de valor $2 . V_{o}$ no conjunto filtro + lâmpadas.

\section{B. Segundo Estágio}

$\mathrm{O}$ circuito e seu equivalente simplificado do segundo estágio são mostrados na Fig. 2.(b) e (e) respectivamente. O estágio é caracterizado pela abertura do interruptor ativo $S$ e pela polarização direta do diodo $D_{5}$. Neste estágio o indutor $L_{\text {Boost }}$ fornece energia para o capacitor $C_{\text {out }}$. A tensão sobre o capacitor $C_{o u t}\left(V_{o}\right)$ é aplicada ao indutor $L_{P 2}$ e refletida pelo acoplamento ao indutor $L_{P}$, resultando em tensão de $-2 . V_{o}$ no conjunto filtro + lâmpadas.

\section{Terceiro Estágio}

$\mathrm{O}$ circuito e seu equivalente simplificado do terceiro estágio são mostrados na Fig. 2.(c) e (f). No terceiro estágio o interruptor $S$ e o diodo $D_{5}$ estão bloqueados. Neste estágio a energia armazenada no circuito ressonante do filtro continua circulando pela lâmpada, completando os estágios de operação do circuito.

As formas de onda teóricas do indutor $L_{b o o s t}$, interruptor ativo $S$, do diodo $D_{5}$ e tensão e corrente no conjunto filtro + lâmpadas de cada estágio da topologia proposta são mostrados na Fig. 2.(g).

\section{IV.EQUAÇÕES RELEVANTES}

Nesta seção são apresentadas as equações dos principais parâmetros de projeto da topologia proposta.

\section{A. Filtro LCC Ressonante}

A topologia proposta é composta de um filtro LCC ressonante de saída, sendo seu capacitor paralelo calculado por (1).

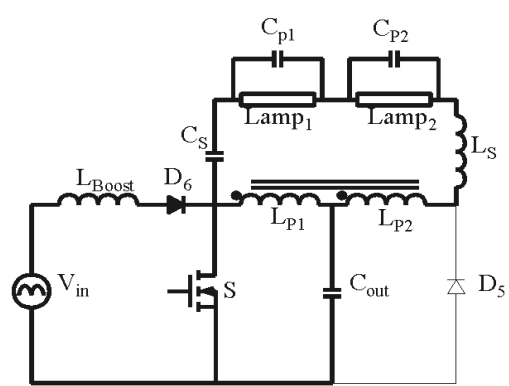

(a) Estágio 1

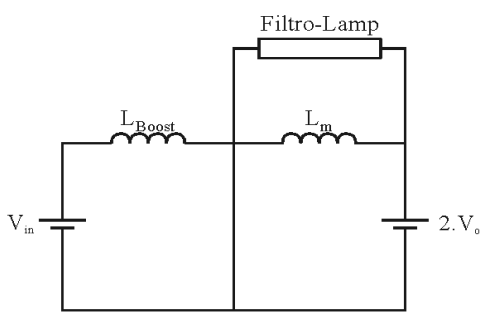

(d) Estágio 1 simplificado

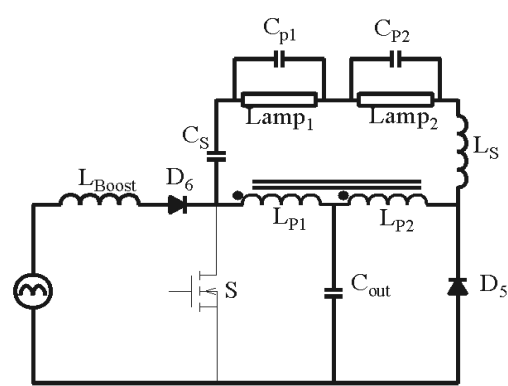

(b) Estágio 2

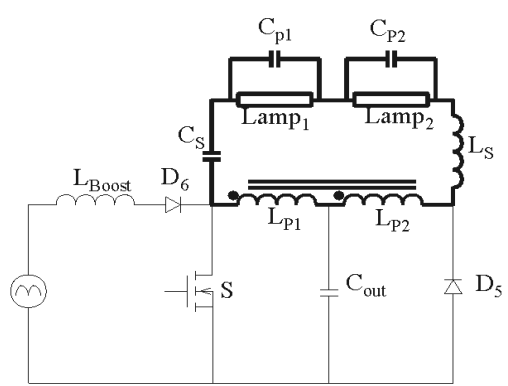

(c) Estágio 3

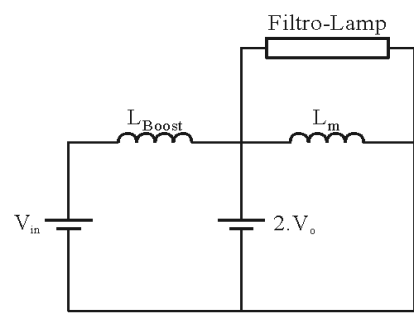

(e) Estágio 2 simplificado

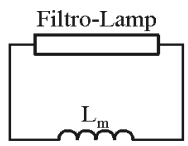

(f) Estágio 3 simplificado

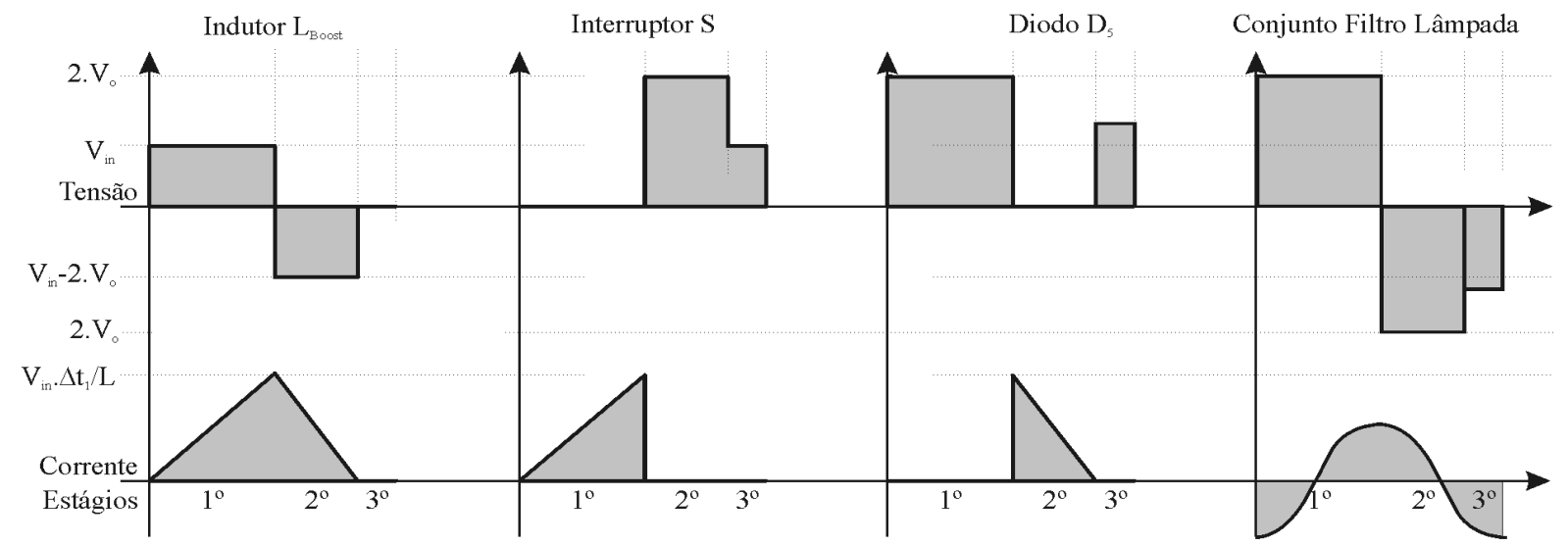

(g) Formas de onda teóricas

Fig. 2. Estágios de operação. 


$$
C_{P}(\theta)=\sqrt{\frac{\left[1+(\tan (\theta))^{2}\right] . P}{\omega^{2} \cdot R \cdot V_{a c}^{2}}-\frac{1}{\omega^{2} \cdot R^{2}}},
$$

onde:

$C_{p}(\theta) \quad$ capacitor paralelo do filtro em função de $\theta$;

$\theta \quad$ ângulo da impedância da entrada;

$P \quad$ potência na lâmpada;

$\omega \quad$ freqüência angular;

$R \quad$ resistência equivalente da lâmpada;

$V_{a c}$ tensão RMS da componente fundamental aplicada ao filtro.

O indutor série é definido por:

$$
L\left(\theta, C_{S}\right)=\frac{R \cdot \tan (\theta)+C_{P}(\theta) \cdot R^{2} \cdot \omega}{\omega \cdot\left[1+\omega^{2} \cdot\left(C_{P}(\theta)\right)^{2} \cdot R^{2}\right]}+\frac{1}{C_{S} \cdot \omega^{2}},
$$

onde:

$C_{s} \quad$ capacitor série suficientemente grande para filtrar a componente contínua da forma de onda da entrada do filtro (arbitrado);

$L\left(\theta, C_{S}\right) \quad$ indutor série do filtro em função de $\theta$ e $\mathrm{C}_{S}$.

$\mathrm{O}$ valor da resistência equivalente em regime permanente para a lâmpada Osram F40W é dado por (3), determinada em [12].

$$
R(P)=25410 \cdot e^{-0,0631 \cdot P}+10126 \cdot e^{-0,263 \cdot P} .
$$

Considerando que a ignição da lâmpada é representada por um elevado valor de resistência equivalente (100R) e sua resistência de operação em regime permanente (R) é definida por (3).

O ângulo $\theta$ a ser utilizado nos cálculos é escolhido de acordo com o gráfico da Fig. 3, definido pela expressão:

$$
P\left(\theta, C_{S}, R\right)=\frac{V_{a c}{ }^{2} \cdot R \cdot A_{1}\left(\theta, C_{S}, R\right)}{R^{2}+\omega^{2} \cdot\left\{A_{2}\left(\theta, C_{S}, R\right)-R^{2} \cdot C_{P}(\theta)\right\}^{2}},
$$

onde:

$$
\begin{aligned}
& A_{1}\left(\theta, C_{S}, R\right)=1+\omega^{2} \cdot C_{P}(\theta)^{2} \cdot R^{2} ; \\
& A_{2}\left(\theta, C_{S}, R\right)=\left[L\left(\theta, C_{S}\right)-\frac{1}{C_{S} \cdot \omega^{2}}\right] \cdot 1+\omega^{2} \cdot C_{P}(\theta)^{2} \cdot R^{2} ;
\end{aligned}
$$

baseado na abordagem da defasagem angular mostrada em [11].

\section{B. Razão Cíclica}

A máxima razão cíclica limite para o modo de condução descontínua é dada por:

$$
D_{\max }=1-\alpha,
$$

onde:

$$
\begin{array}{ll}
D_{\max } & \text { máxima razão cíclica; } \\
\alpha & V_{P} / 2 . V_{C} ; \\
V_{P} & \text { tensão de pico da entrada; } \\
V_{C} & \text { tensão no capacitor } C_{\text {out }} .
\end{array}
$$

Para obter o modo de condução descontínuo deve-se escolher uma razão cíclica menor que $D_{\max }$, portanto:

$$
D_{d}<1-\alpha \text {. }
$$

\section{Indutância Boost}

A indutância boost é definida pela seguinte expressão

$$
L_{B o o s t}=\frac{V_{P}^{2}}{2 \cdot \pi \cdot f \cdot P} \cdot \frac{(1-\alpha)^{2}}{\alpha} \cdot Y(\alpha),
$$

onde:

$$
\begin{aligned}
& Y(\alpha)=-2-\frac{\pi}{\alpha}+\frac{2}{\alpha \cdot \sqrt{1-\alpha^{2}}} \cdot\left[\frac{\pi}{2}+\tan ^{-1}\left(\frac{\alpha}{\sqrt{1-\alpha^{2}}}\right)\right] ; \\
& P_{\text {out }} \quad \text { potência de saída; } \\
& f \quad \text { freqüência de operação. }
\end{aligned}
$$

\section{Correntes de entrada}

A corrente eficaz de entrada pode ser definida por:

$$
I_{i n, R M S}=\frac{P}{V_{i n} \cdot \eta},
$$

onde:

$$
\begin{array}{ll}
P & \text { potência de entrada; } \\
V_{\text {in }} & \text { tensão RMS de entrada. }
\end{array}
$$

A corrente de pico no indutor Boost é definida por:

$$
i_{\text {Boost }, \text { Pico }}=\frac{V_{\text {in }} \cdot D}{L_{\text {Boost }} \cdot f} .
$$

\section{E. Corrente no Push-Pull}

A corrente de pico no push-pull é dado por:

$$
i_{P P, \text { pico }}=i_{L, \text { pico }}+i_{\text {mag }} \text {, }
$$

onde:

$$
\begin{array}{ll}
i_{\text {Lpico }} & \text { corrente de pico na lâmpada; } \\
i_{\text {mag }} & \text { corrente na indutância magnetizante. }
\end{array}
$$

\section{F. Avaliação dos Esforços}

A máxima corrente no interruptor é dado por:

$$
i_{S}=i_{\text {in }, \text { pico }}+i_{P P, p i c o}+i_{\text {mag }} \text {. }
$$

A tensão sobre o interruptor é dada por:

$$
V_{S}=2 \cdot V_{C} \text {. }
$$

A corrente no diodo $\mathrm{D}_{5}$ é dada por:

$$
i_{D 5, R M S}=i_{\text {mag, RMS }} \text {. }
$$

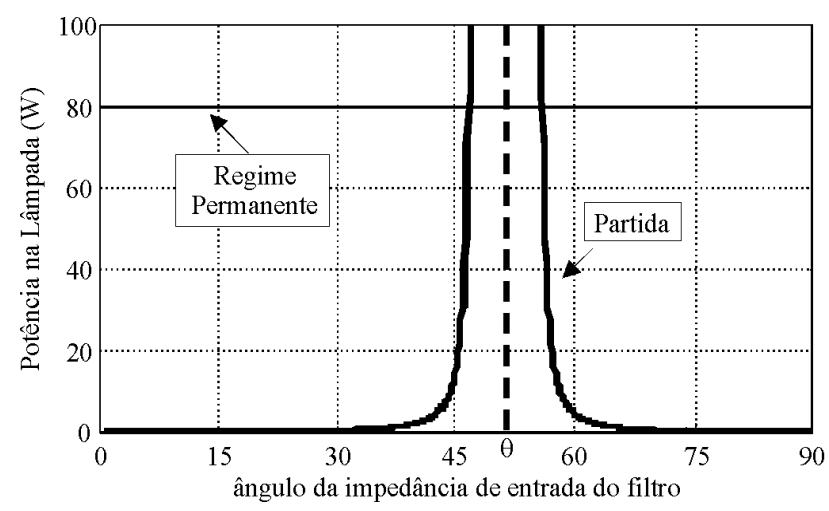

Fig. 3. Gráfico da potência na lâmpada em função do ângulo da impedância de entrada do filtro $L C C$ ressonante 


\section{CÁLCULOS DO PROTÓTIPO}

Os cálculos do protótipo foram feitos para os seguintes dados de entrada:

Tensão de entrada:

Carga:

Frequiência de operação:

$110 \mathrm{~V}_{\mathrm{RMS}}, 60 \mathrm{~Hz}$;

2 lâmpadas "T12" de 40W;

$50 \mathrm{kHz}$.

\section{A. Filtro LCC Ressonante}

Através da expressão (4) podem ser obtidos os gráficos das potência na lâmpada em regime e na ignição, mostrados na Fig. 3, considerando:

Tensão de pico a pico de entrada no filtro: $600 \mathrm{~V}$;

Calculando a tensão RMS da componente fundamental da tensão aplicada ao conjunto filtro lâmpada.
Vac:
$270 \mathrm{~V}$.

Calcula-se pela equação (3) a resistência equivalente da lâmpada Osram F40W, obtendo

$R$ :

$340 \Omega$.

Adota-se

$C_{S}$ :

$150 \mathrm{nF}$.

A partir dos dados definidos acima é possível construir o gráfico mostrado na Fig. 3 e escolher o ângulo que possibilita um elevado ganho na partida:

$\theta$ :

$49^{\circ}$

Considerando o ângulo $\theta$, determinado graficamente os valores de $C_{P}$ e L que são respectivamente calculados através de (1) e (2), fornecendo os seguintes resultados:

$$
\begin{array}{lr}
C_{P}: & 4 \mathrm{nF} ; \\
L: & 2,6 \mathrm{mH} .
\end{array}
$$

\section{B. Razão Cíclica Máxima}

Para o cálculo da razão cíclica têm-se

$V_{P} \quad 155 \mathrm{~V}$;

$V_{C} \quad 150 \mathrm{~V}$

de (5) obtém-se

$\begin{array}{ll}\alpha & 0,52 ; \\ D_{\max } & 0,48 .\end{array}$

\section{Indutância Boost}

O cálculo da indutância boost é dado por (7) e (8) que resulta

$$
L_{\text {Boost }}
$$

$640 \mu \mathrm{H}$.

D. Correntes de Entrada

As correntes de entrada são calculadas pelas expressões

(9) e (10) obtendo-se os seguintes valores:
$I_{\text {in, RMS }}$
$808 \mathrm{~mA}$;
$I_{\text {Boost,Pico }}$
2,342A.

E. Corrente no Push-Pull

A corrente no transformador push-pull é calculada pela expressão (11)
$I_{L, p i c o}$
$343 \mu \mathrm{A}$
$I_{\text {mag }}$
$963 \mu \mathrm{A}$
$I_{P P \text {,pico }}$
$1,3 \mathrm{~mA}$.

F. Avaliação dos Esforços

A máxima corrente no interruptor para o pior caso é dada por (12):
$I_{S}$
$3,65 \mathrm{~A}$.

A tensão sobre o interruptor e sobre o diodo é dada por (13):

$V_{S} \quad 300 \mathrm{~V}$.

A corrente no diodo $\mathrm{D}_{5}$ é dada por (14):

$I_{D 5, R M S}$

$300 \mathrm{~mA}$.

\section{RESULTADOS EXPERIMENTAIS}

A Fig. 4 mostra o protótipo da topologia proposta construído com base nas especificações de projeto apresentadas abaixo:

- Tensão de alimentação: $V_{i n}=110 \mathrm{~V}_{\mathrm{RMS}}, 60 \mathrm{~Hz}$;

- Potência de saída: $64 \mathrm{~W}$;

- Freqüência de comutação: $f=50 \mathrm{kHz}$;

- Razão cíclica: $D=0,48$.

Os componentes utilizados para a montagem do protótipo são os seguintes:

- $L_{\text {Boost }}: 500 \mu \mathrm{H}, 50$ espiras com núcleo EE 24/12 IP6 Thornton;

- $L_{P 1}, L_{P 2}: 3 \mathrm{mH}, 120$ espiras com núcleo EE30/14 IP6Thornton;

- $D_{1}-D_{2}: 1 \mathrm{~N} 4007$

- $D_{5}, D_{6}$ : UF4007;

- $S$ : IRF740 (International Rectifier);

- $C_{\text {out }}: 110 \mu \mathrm{F} / 250 \mathrm{~V}$ (eletrolítico).

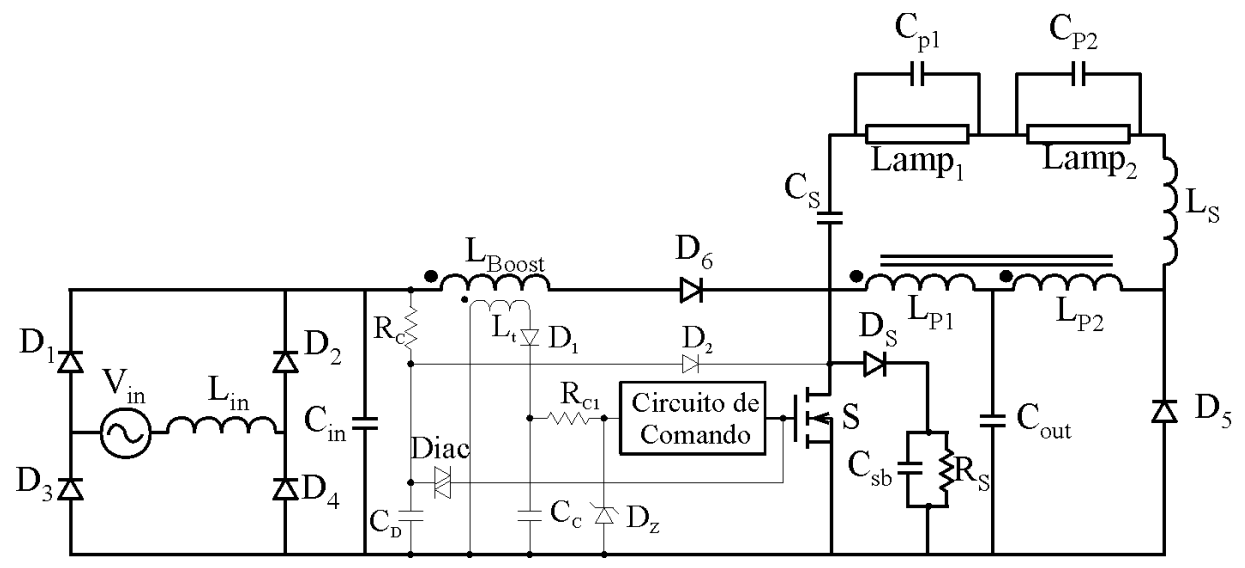

Fig. 4. Protótipo implementado 
O filtro EMI possui os seguintes componentes:

- $L_{i n}$ : 1,4mH, 150 espiras com núcleo EE20/10 IP6Thornton.

O filtro ressonante de saída tem os seguintes componentes:

- $L_{\text {out }}:$ 2,7 mH, 150 espiras com núcleo EE20/10 IP6Thornton;

- $C_{S}: 150 \mathrm{nF} / 250 \mathrm{~V}$ (polipropileno);

- $C_{P 1}, C_{P 2}: 3,6 \mathrm{nF} / 2 \mathrm{kV}$ (polipropileno).

Foram utilizadas duas lâmpadas fluorescentes tubulares Osram F40W:

- $D_{S}:$ UF4007;

- $C_{S b}: 10 \mathrm{nF}$;

- $R_{S}: 12 \mathrm{k} \Omega$

As formas de onda obtidas experimentalmente estão ilustradas nas Fig. 5 a Fig. 14.

A corrente e tensão de entrada, na Fig. 5, mostram o alto fator de potência e a THD reduzido da corrente.

Nas Fig. 6 e Fig. 7 são mostrados as formas de onda da corrente no indutor $L_{\text {Boost }}$ em diferentes escalas de tempo, comprovando o modo de condução descontínua.

A tensão e corrente na chave $S$ e no diodo $D_{5}$ são mostradas respectivamente nas Fig. 8 e Fig. 9. Na Fig. 10 podese observar a reduzida ondulação na tensão do capacitor $C_{\text {out }}$, não havendo influência considerável na variação do fluxo luminoso das lâmpadas, como pode ser concluído através da envoltória da corrente na lâmpada, Fig. 12, o que resulta em um fator de crista adequado.

A Fig. 11 mostra a forma de onda da tensão e corrente no transformador push-pull, onde pode-se verificar a forma de onda quadrada característica destes conversores.

A tensão e a corrente em alta freqüência em uma das lâmpadas fluorescentes são ilustradas na Fig. 13, observando se que as mesmas se aproximam de uma forma de onda senoidal. Na Fig. 14 é mostrada a tensão de partida da lâmpada, na qual pode ser observada a partida instantânea.

Analisando-se os dados obtidos experimentalmente das formas de onda da topologia proposta, foram obtidos os seguintes resultados:

- rendimento $\eta(\%)=87 \%$;

- fator de potência de $\mathrm{fp}=0,99$;

- taxa de distorção harmônica da corrente de entrada THD $=13,75 \%$;

- fator de crista da corrente em uma das lâmpadas de 1,5;

- potência em uma das lâmpadas $\mathrm{P}=32 \mathrm{~W}$.

A potência obtida na lâmpada em alta freqüência produz um fluxo luminoso equivalente a uma lâmpada fluorescente "T12" com potência nominal de $40 \mathrm{~W}$ em baixa freqüência.

\section{VII.CONCLUSÃO}

O artigo apresenta uma nova topologia de reator eletrônico para lâmpadas fluorescentes com alto fator de potência e baixo custo. A topologia proposta possui dois conversores operando em cascata, empregando apenas um interruptor ativo. O primeiro é o conversor boost empregado na correção do fator de potência e o segundo é o conversor push-pull, que alimenta a carga (filtro e lâmpadas) em alta freqüência.
O conversor boost opera no modo de condução descontínua, dispensando o controle de corrente no indutor Boost, possibilitando a operação do circuito em malha aberta.

$\mathrm{O}$ conversor push-pull permite empregar a energia armazenada nos enrolamentos para viabilizar a comutação espontânea do diodo $\mathrm{D}_{5}$, dispensando um dos interruptores seu circuito de comando.

O interruptor compartilhado $(S)$ pelos dois conversores não requer circuito de comando isolado, simplificando ainda mais o circuito de comando, porém possui uma corrente elevada inerente ao compartilhamento.

Desta forma, esta topologia reúne simplicidade e eficiência na obtenção de um reator eletrônico com alto fator de potência, alta eficiência luminosa e custo reduzido.

Os resultados experimentais demonstram que a topologia proposta encontra-se dentro dos padrões mundiais, no que diz respeito a correção do fator de potência, rendimento, e fator de crista da corrente.

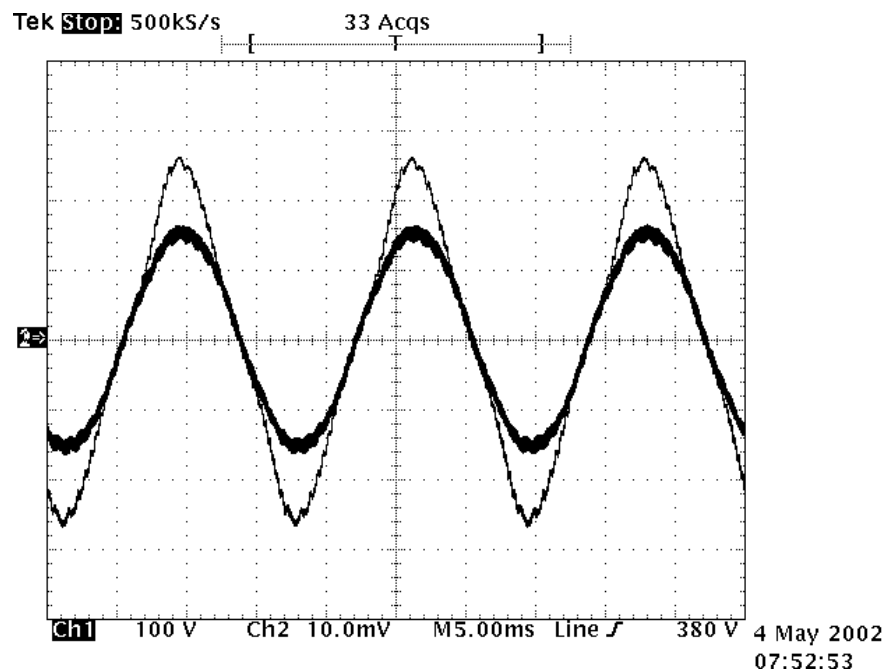

Fig. 5. Tensão e corrente na entrada (50V/div, 1A/div e $5 \mathrm{~ms} / \mathrm{div})$

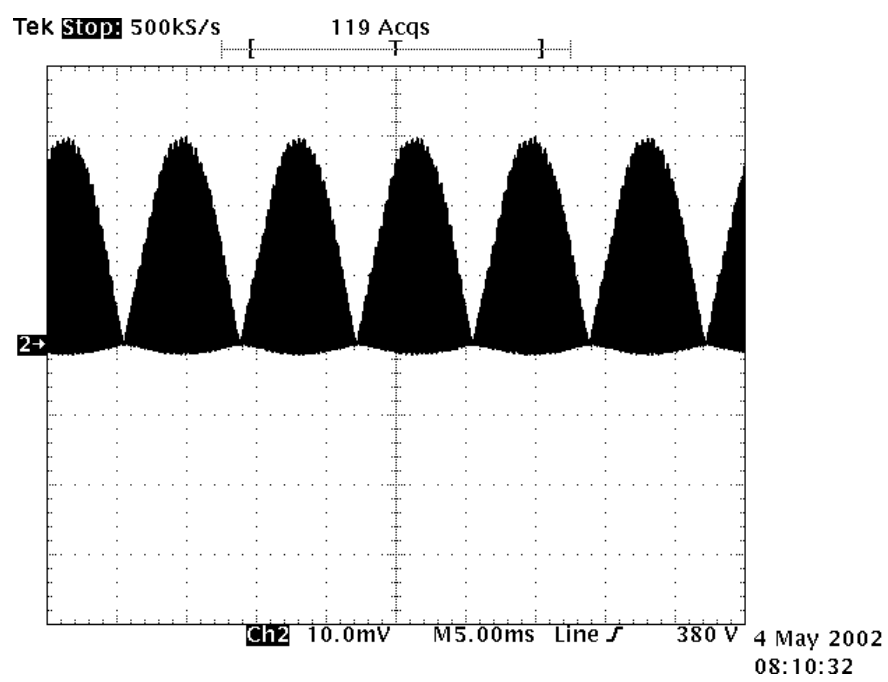

Fig. 6. Corrente no indutor boost em baixa frequiência. (1A/div e 5ms/div) 


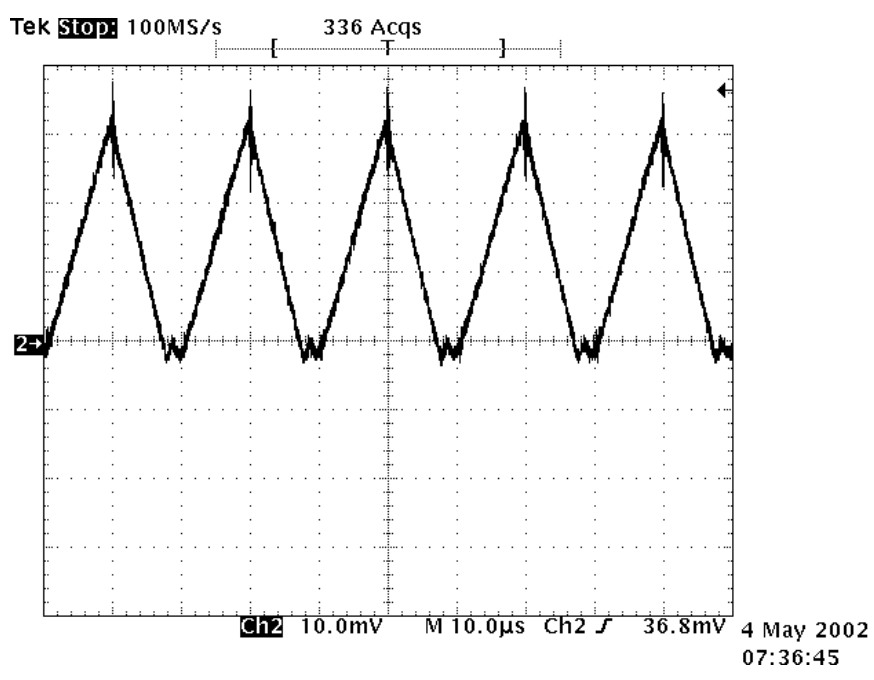

Fig. 7. Corrente no indutor boost em alta freqüência (1A/div e $10 \mu \mathrm{s} / \mathrm{div})$

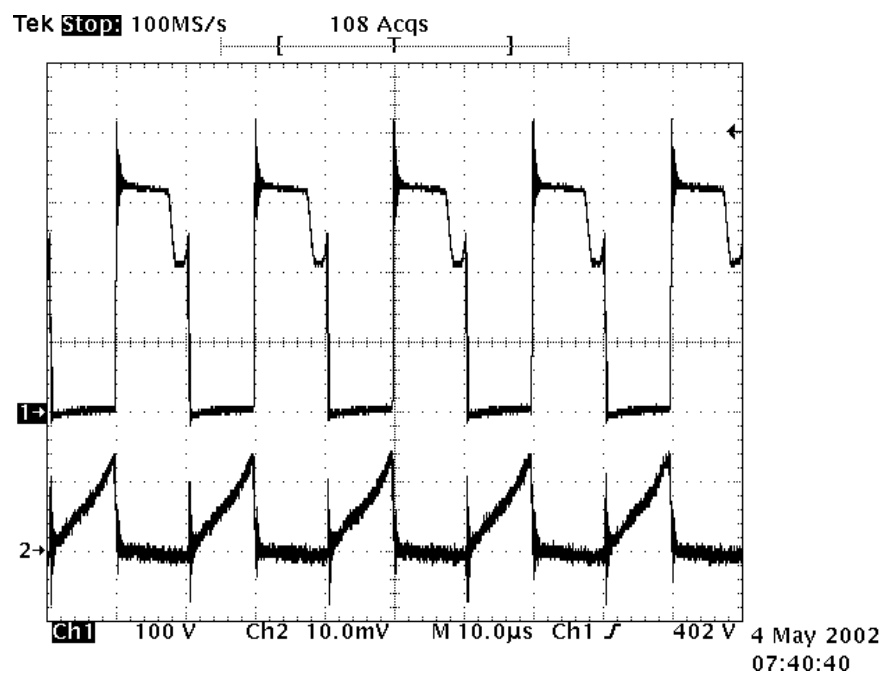

Fig. 8. Tensão e corrente no interruptor (100V/div, 5A/div e 10 $\mu \mathrm{s} / \mathrm{div})$

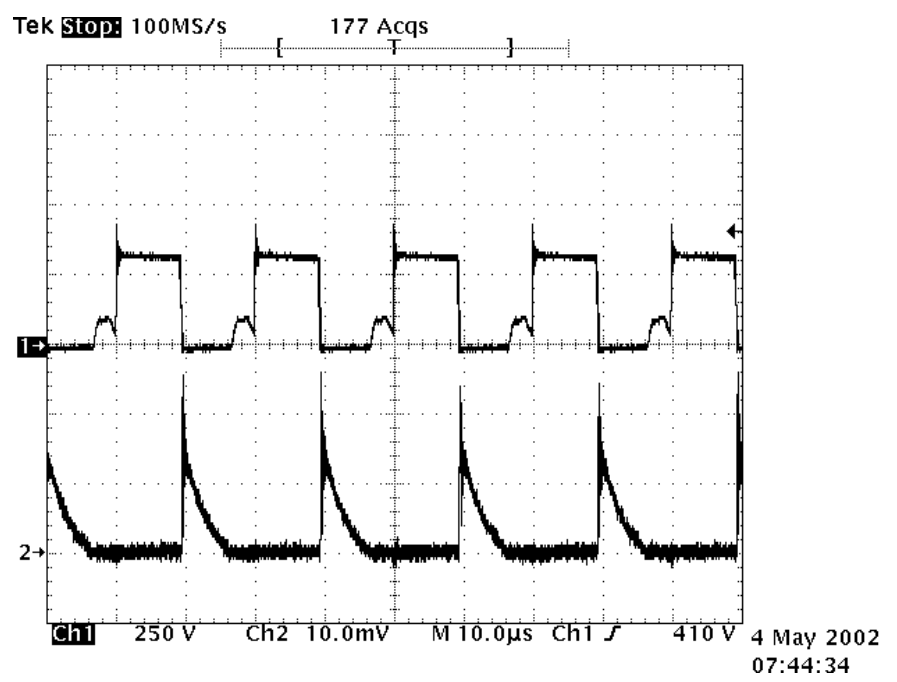

Fig. 9. Tensão e corrente no diodo $D_{5}$ (250V/div, $5 \mathrm{~A} / \mathrm{div}$ e $10 \mu \mathrm{s} / \mathrm{div})$

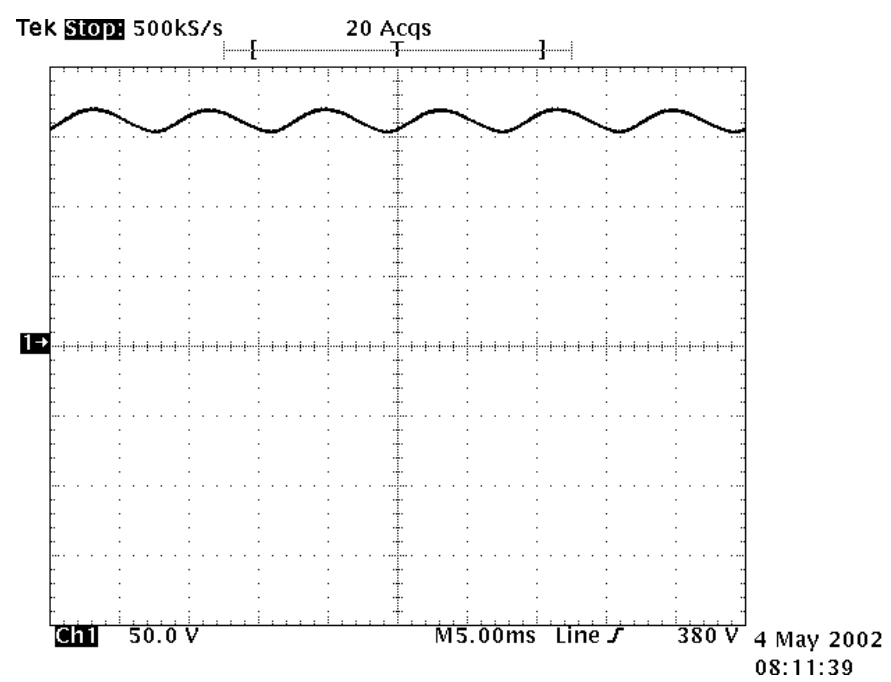

Fig. 10. Tensão no capacitor Cout (50V/div e $5 \mathrm{~ms} / \mathrm{div})$

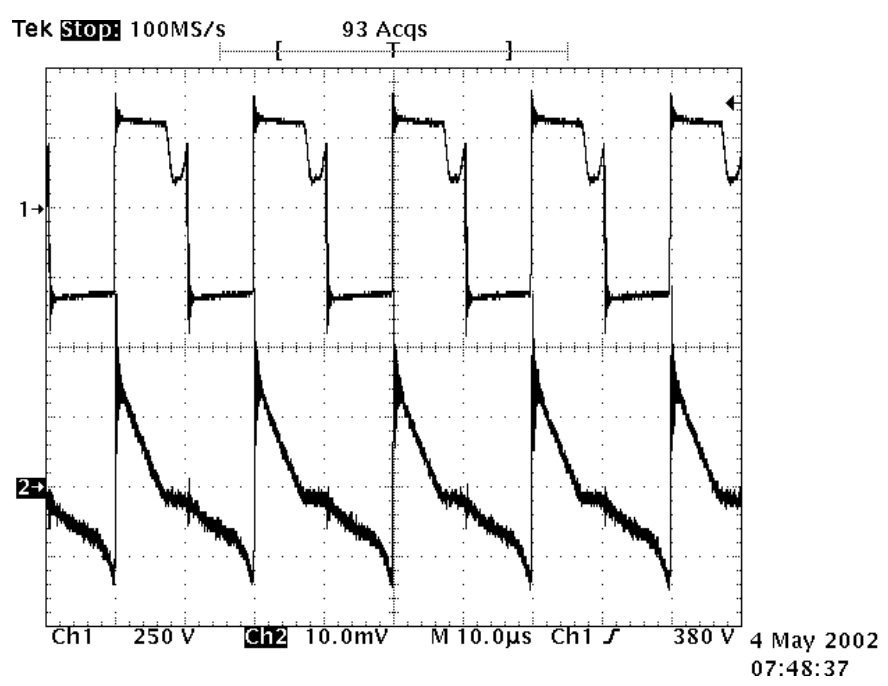

Fig. 11. Tensão e corrente no transformador Push-Pull $(250 \mathrm{~V} / \mathrm{div}, 500 \mathrm{~mA} / \mathrm{div}$ e $10 \mu \mathrm{s} / \mathrm{div})$

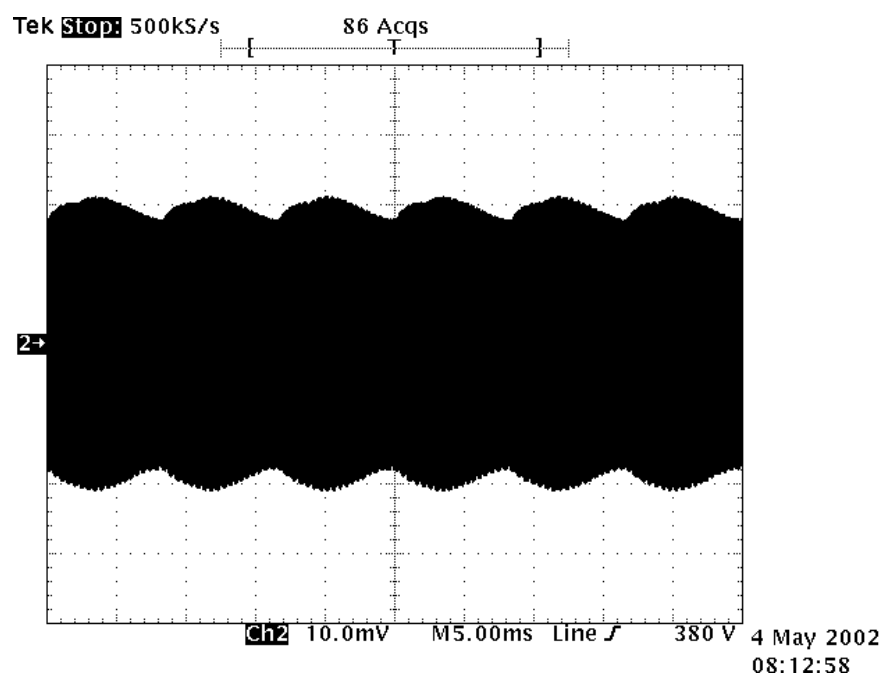

Fig. 12. Envoltória da corrente na lâmpada $(200 \mathrm{~mA} / \mathrm{div}$ e $5 \mathrm{~ms} / \mathrm{div})$ 


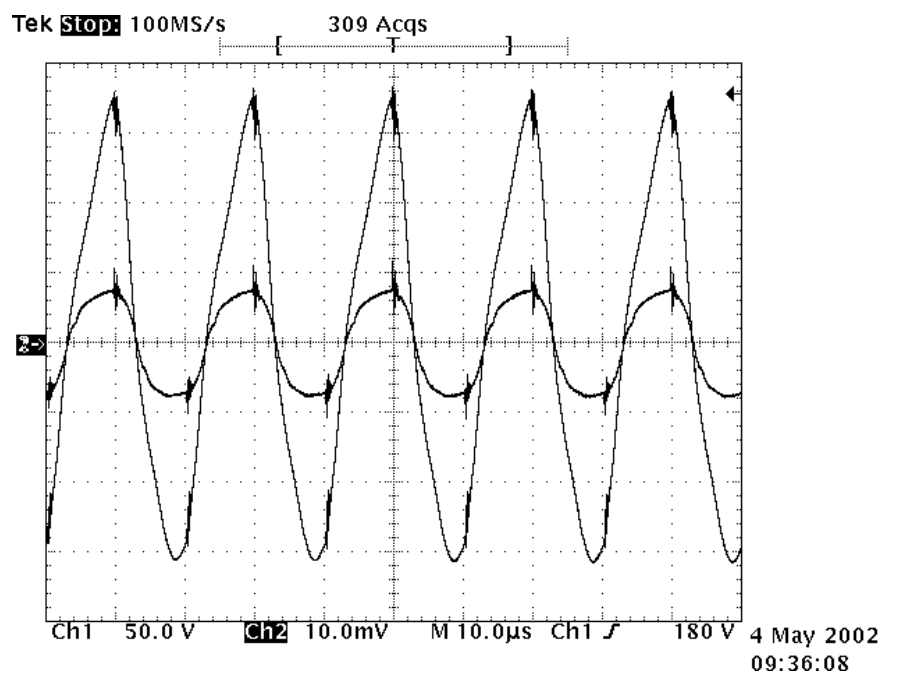

Fig. 13. Forma de onda da tensão e corrente na lâmpada em alta frequiência $(50 \mathrm{~V} / \mathrm{div}, 500 \mathrm{~mA} / \mathrm{div}$ e $10 \mu \mathrm{s} / \mathrm{div})$

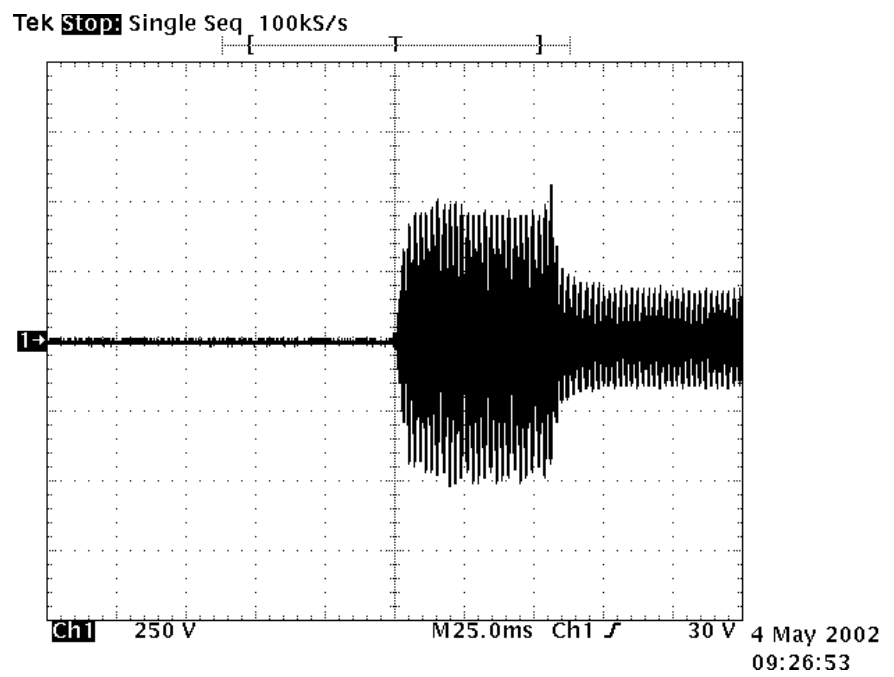

Fig. 14. Forma de onda da tensão na partida da lâmpada (250V/div e 25ms/div)

\section{AGRADECIMENTOS}

Os autores agradecem à THORNTON INPEC pelo apoio com núcleos utilizado nas montagens, à OSRAM do Brasil pelo fornecimento das lâmpadas utilizadas nos ensaios, à CAPES e CNPq pelo apoio financeiro.

\section{REFERÊNCIAS BIBLIOGRÁFICAS}

[1] R. N. do Prado, S. A, Bonaldo and D. S. Greff, “A High Power Factor Flyback-Half-Bridge Electronic Ballast with Dimming Feature," IEEE IAS'98, record.

[2] W. R. Alling, "Important Design Parameters for SolidState Ballasts," IEEE Transactions on Industry Applications, vol. 25, no. 2, pp. 203-207, march/abril 1989.

[3] T. H. Yu and L. M. Wu. "Comparisons Among SelfExcited Parallel Resonant, Series Resonant and CurrentFed Push-Pull Electronic Ballasts", IEEE Applied Power Electronic Conference, pp. 421-426, 1994.
[4] E. E. Hammer, "High Frequency Characteristics of Fluorescent Lamps up to $500 \mathrm{kHz}$ ", Journal of the Illuminating Engineering Society, pp.56-61, Winter 1987.

[5] E. E. Hammer and T. K. McGowan, "Characteristics of Various F40 Fluorescent Systems at $60 \mathrm{~Hz}$ and High Frequency," IEEE Transactions on Industry Applications, vol. 21, no. 1, pp. 11-16, 1985.

[6] R. N. Prado, S. A. Bonaldo, M. C. Moreira, D. L. R. Vidor. "Eletronic Ballast with a High Power for Fluorescent Lamp”. IEEE PESC'96, Record, pp. 1215120.

[7] R. N. Prado, S. A. Bonaldo, D. S. Greff, and F. E. Bisogno. "A Unity Power Factor Eletronic Ballast for Fluorescent Lighting, " IEEE IAS'97 Annual Meeting Records, pp. 2366-2371.

[8] J. A. Sierra and W. Kaiser. "Comparison of Fluorescent Lamp Stabilization Methods in the Current-Fed PushPull Inverter," IEEE Trans. Industry Application, vol. 36, no. 1, January/February 2000.

[9] R. R. Verderber, O. C. Morce, and F. M. Rubinstein, "Performance of Electronic Ballast and Control with 34 and 40 watt F40 Fluorescent Lamps," IEEE Transactions on Industry Applications, vol. 25, no. 6, pp. 1049-1059, November/December 1989.

[10] M. K. Kazierczuck and W. Szaraniec, "Electronic Ballast for Fluorescent Lamps," IEEE Transactions on Power Electronics, vol. 8, no. 4. pp. 386-395, October 1993.

[11] Y. Takahashi, M. Kamata, K. Shimizu, "Efficiency Improvement of Electronic Ballast," IEEE IAS'97, Record.

[12] M. Cervi, A. R. Seidel, F. E. Bisogno, R. N. Prado, "Fluorescent Model Based on the Equivalent Resistance Variation," IEEE IAS'02, record.

\section{DADOS BIOGRÁFICOS}

Fábio Ecke Bisogno, nascido em 07/04/1973 em Santa Maria (RS) é engenheiro eletricista (1999) e mestre (2001) pela Universidade Federal de Santa Maria, onde é doutorando no Programa de Pós-graduação em Engenharia Elétrica. Atualmente é pesquisador no Grupo de Estudo e Desenvolvimento de Reatores Eletrônicos (GEDRE) nesta mesma universidade. Suas áreas de interesse são lâmpadas fluorescentes, reatores eletrônicos, sistemas dimerizáveis e fontes de alimentação.

Álysson Raniere Seidel, nascido em 01/08/1975 em São Pedro do Sul (RS) é engenheiro eletricista (1999) pela Universidade Federal de Santa Maria, onde é doutorando no Programa de Pós-graduação em Engenharia Elétrica. Atualmente é pesquisador no Grupo de Estudo e Desenvolvimento de Reatores Eletrônicos (GEDRE) nesta mesma universidade. Suas áreas de interesse são lâmpadas fluorescentes, reatores eletrônicos, sistemas dimerizáveis e eficiência luminosa. 
Tiago Bandeira Marchesan, nascido em 09/12/1980 em Santa Maria (RS) estudante de Engenharia Elétrica na Universidade Federal de Santa Maria. Atualmente é pesquisador no Grupo de Estudo e Desenvolvimento de Reatores Eletrônicos (GEDRE) nesta mesma universidade. Suas áreas de interesse são lâmpadas fluorescentes, reatores eletrônicos, fontes de alimentação e sistemas dimerizáveis.

Ricardo Nederson do Prado, nascido em 22/04/1960 em Itapiranga (SC) obteve o título de engenheiro eletricista pela Universidade Federal de Santa Maria, em 1984, e os títulos de mestre e doutor em engenharia elétrica pela Universidade Federal de Santa Catarina em 1987 e 1993, respectivamente. Entre 1987 e 1992 foi professor assistente junto ao departamento de eletrônica da Universidade Federal de Minas Gerais. A partir de 1993 é professor adjunto no departamento de eletrônica e computação da Universidade Federal de Santa Maria. É membro do IEEE, da SBA e membro fundador da SOBRAEP. Atualmente é responsável pelo grupo de estudo e desenvolvimento de reatores eletrônicos (GEDRE) e coordenador do programa de Pós-graduação da Universidade Federal de Santa Maria. Suas áreas de interesse são sistemas dimerizáveis e reatores eletrônicos para lâmpadas fluorescentes e de alta pressão, qualidade de energia e eficiência luminosa. 